\title{
Evaluation and analysis of an enhanced hybrid wireless mesh protocol for vehicular ad hoc network
}

\author{
Amal A. Eltahir ${ }^{1 *}$, Rashid A. Saeed ${ }^{1}$, Amitava Mukherjee ${ }^{2}$ and Mohammad Kamrul Hasan ${ }^{3}$
}

\begin{abstract}
This paper presents a new clustering mechanism for the multi-hop relay in vehicular communication. In the proposed mechanism, the cluster-head selection is implemented using the enhanced hybrid wireless mesh protocol (E-HWMP) protocol, which is based on the IEEE802.11p and IEEE802.11s standards. Then, multi-hop routing is designed for VANET in Wireless Mesh Networks (WMNs). Therefore, the main aim of this study is to enhance the integration of VANET with a cellular network (i.e., LTE). As a result, the coverage can be extended. The proposed E-HWMP mechanism is evaluated by the NS2 simulator, and the VanetMobiSim is used as a mobility generator. The proposed E-HWMP mechanism is compared to the existing mechanism based on the packet's delivery ratio, packet loss, throughput, overhead, and average end-to-end delay. The results demonstrate that the proposed E-HWMP mechanism outperforms the existing clustering mechanism.
\end{abstract}

Keywords: Vehicular ad hoc network (VANET), HWMP, Gateway selection, LTE, NS-2.35

\section{Introduction}

Recently, vehicular ad hoc network (VANET) has attracted the attention of academia as well as industries due to its promising applications. VANET is categorized based on vehicles' locations and their speeds and is classified into two major categories namely, Vehicle-toVehicle (V2V) and Vehicle-to-Infrastructure (V2I) communication [1].

VANET is a dynamic network topology which consists of high-speed vehicles that moves in a specific direction. There are many services (e.g., driver aid, collision alarm, traffic information, and tracking) provided by VANET in order to improve road safety and transportation efficiency [2]. There are many obstacles faced by the vehicles' network such as frequency interruption of network connectivity and limited coverage area. As a result, to meet the road-side safety, transportation communication efficiency, and the demands for

\footnotetext{
* Correspondence: amaleltahir@hotmail.com

${ }^{1}$ Electronics Engineering School, Sudan University of Science and Technology (SUST), Khartoum, Sudan

Full list of author information is available at the end of the article
}

the various entertainment applications for vehicles, the selection of the appropriate protocol is strongly recommended. Our paper addresses the above identified issues by the following key contributions:

1) Design a new clustering mechanism for the multihop relay in vehicular communication.

2) The use of E-HWMP protocol to implement the proposed mechanism.

3) Enhanced vehicular network formulation with reducing signalling and overhead traffic.

4) Evaluate the proposed scheme under multi-metrics design parameters for overall throughput.

The rest of the paper is organized as follows: Section 2 highlights the related works. The proposed mechanism is designed and discussed in Section 3. Section 4 evaluates the performance of the proposed mechanism. The paper is concluded in Section 5.

\section{Related works}

Gateway vehicle selection and management is the prominent issue in the mechanism of multi-hop relay based 
on the Integrated VANET-cellular networks Heterogeneous Wireless Network. This is due to the nonsimilarity characteristics in vehicles, which lead to inability of the gateway to access. To solve this problem, one of the proposed solutions is to assemble them in groups (clusters) based on vehicles with similar characteristics and elect a cluster head $(\mathrm{CH})$ for each group (cluster), which becomes the gateway node. Vehicle clustering is promising to resolve this issue, where cluster-head selection depends only on the geographical location of vehicles. In consequence, the cluster head of the cluster does not have the basic standards to manage the VANET groups, so that it enables them to connect with the cellular network.

The proposed mechanism stated in [3], Simplified Gateway Selection Scheme (SGSS), enabled vehicles to connect to the Internet and other mobile services continuously; it described two types of vehicles: vehicles within the coverage area had a dual interface and communicated directly to the UMTS base station transceiver. UTRAN and vehicles that move along the road looking for an associate to be used to connect to the UMTS base station transceiver had a single interface. Vehicles received different signals from the base station transceiver. This mechanism showed lower reliability in terms of availability. In addition, the researcher did not take into account the propagation delay between the relay request message and the relay response message.

A Cluster-based Multi-metric, adaptive mobile Gateway Management mechanism (CMGM) was presented, where two kinds of vehicles were described [4], namely, vehicles equipped with IEEE $802.11 \mathrm{p}$ and vehicles with UTRAN interfaces. It was discovered that the mechanism allowed the small number of vehicles $(\mathrm{GW})$ with IEEE $802.11 \mathrm{p}$ to connect to the $3 \mathrm{G}$ networks and the UTRAN interfaced vehicles used this GW to connect to the 3GPP networks. The clustering scheme utilized to cluster GWc was based on the direction of movement, UMTS-RSS and IEEE 802.11p transmission ranges. If the GWc was closest to the middle of the cluster, then $\mathrm{CH}$ was elected in a cluster. However, the suggested mechanism in [2] was complex in terms of preserving the clusters for the fast-moving environment such as in VANET. In addition, the process of selecting the $\mathrm{CH}$ depends mainly on the geographical location of the vehicle. The result showed that the $\mathrm{CH}$ did not have the basic criteria for managing the cluster and enabling them to connect to the cellular network. In addition, the process of forming a cluster and the selection of the $\mathrm{CH}$ consumed more time compared with data exchange time.

In [5], an optimized hybrid wireless mesh protocol had been suggested to construct a routing protocol over
VANET. A real-time packet loss estimation was utilized as an additional parameter to the parameters used in [4] (UMTS received signal strength, link stability, and available relay capacity) to elect an efficient mobile gateway node for the transfer of packets without losing any of them.

The authors in [6] suggested a Cluster-based Multimetric, new Simplified Gateway Selection Scheme (SGSS), where the received signal strength (RSS) threshold was utilized as a cluster formation index considering the UMTS cell boundary. The Gateway had been selected based on the UMTS received signal strength, link stability, and available relay capacity. An enhanced hybrid wireless mesh protocol (E-HWMP) protocol was suggested to construct multi-hop routing through vehicular ad hoc networks (VANET).

The integration of IEEE802.11p VANETs with 3GPP LTE for seamless data connectivity had been proposed in heterogeneous wireless network architecture [7]. Issues on $\mathrm{CH}$ based on multicasting and QoS was described in this study. The approach had suggested the mechanism for the selection of an adaptive multi-metric $\mathrm{CH}$ to manage VANET sub-clusters and to provide multimedia communication services over spatially apart vehicular clusters. As expected from the effective cluster-head selection mechanism, there was a suggested approach for a virtual two-hop overlay mesh-based shared tree for lower level VANET multicasting. The results showed that the integrated system had an acceptable output in terms of throughput and end-end delay for the LTE network.

The mechanism proposed in [8] was to connect vehicular ad hoc networks to the infrastructure networks. The work included an experiential estimate of relay vehicle utilization when UMTS Node B was far away from the source vehicle. This scheme allowed the source vehicle to find a relay vehicle before exiting the coverage zone of the RSU. When implementing this mechanism, the achieved result was the extension of the network coverage zone.

In [9], the authors proposed spatial reusability-aware single-path routing (SASR) and any path routing (SAAR) protocols and compare them with existing single-path routing and any path routing protocols, respectively. The evaluation results show that our protocols significantly improve the end-to-end throughput compared with existing protocols. Specifically, for single-path routing, the median throughput gain is up to $60 \%$, and for each source-destination pair, the throughput gain is as high as 5.3×; for any path routing, the maximum perflow throughput gain is $71.6 \%$, while the median gain is up to $13.2 \%$.

In [10] the authors propose a cross-layer distributed algorithm called interference-based topology control 
algorithm for delay-constrained (ITCD) MANETs with considering both the interference constraint and the delay constraint, which is different from the previous work. They focus on the delay-constrained topology control problem and take into account delay and interference jointly. The transmission delay, contention delay, and the queuing delay are taken into account in the proposed algorithm. Moreover, the impact of node mobility on the interference-based topology control algorithm is investigated, and the unstable links are removed from the topology. The simulation results show that ITCD can reduce the delay and improve the performance effectively in delay-constrained mobile ad hoc networks.

In [11], the authors propose a reliable multicast protocol, called CodePipe, with energy efficiency, high throughput, and fairness in lossy wireless networks. Building upon opportunistic routing and random linear network coding, CodePipe can not only eliminate coordination between nodes but also improve the multicast throughput significantly by exploiting both intra-batch and inter-batch coding opportunities. In particular, four key techniques, namely, LP-based opportunistic routing structure, opportunistic feeding, fast batch moving, and inter-batch coding, are proposed to offer significant improvement in throughput, energy efficiency, and fairness. Moreover, the authors design an efficient online extension of CodePipe such that it can work in a dynamic network where nodes join and leave the network as time progresses. The proposed protocol evaluated on ns2 simulator by comparing with other two state-of-art multicast protocols, MORE and Pacifier. Simulation results show that CodePipe significantly outperforms both of them.

In [12], the authors present a directional routing and scheduling scheme (DRSS) for green vehicle DTNs by using Nash Q-learning approach that can optimize the energy efficiency with the considerations of congestion, buffer, and delay. Their scheme solves the routing and scheduling problem as a learning process by geographic routing and flow control toward the optimal direction. To speed up the learning process, their scheme uses a hybrid method with forwarding and replication according to traffic pattern. The DRSS algorithm explores the possible strategies and then exploits the knowledge obtained to adapt its strategy and achieve the desired overall objective when considering the stochastic non-cooperative game in online multicommodity routing situations. The simulation results of a vehicular DTN with predetermined mobility model show DRSS achieves good energy efficiency with learning ability, which can guarantee the delivery ratio within the delay bound.
In [13], the authors study the heterogeneous media provision in peer-to-peer (P2P)-based vehicular networks and develop fully dynamic service schemes with the goals of maximizing the total usersatisfaction and achieving a certain amount of fairness. We first construct a general user-satisfaction model according to the network transmission mechanism, as well as different media delay-satisfaction characteristics. Then, the media service was formulated as an optimization problem and proposes a joint content dissemination and cache update scheme. Also, the exact steps to achieve the optimal solution at equilibrium, given the user-satisfaction function, were provided. Furthermore, the proposed service scheme was extended by addressing the fairness problem. Unlike prior works that target at bandwidth or demand fair, a media-aware satisfaction-fairness strategy, which is aware of the characteristic of usersatisfaction and media content and ensures max-min satisfaction-fairness sharing among multiple vehicles, was proposed. It is worth noting that both schemes are designed in a distributed manner, which is amenable to online implementation for vehicle networks. In addition, extensive simulation results that demonstrate the effectiveness of our proposed schemes were provided.

In [14], the authors present overview characteristics of cloud computing and introduces the basic concepts of vehicular networks. An architecture for multimedia cloud computing is proposed to suit subscription service mechanisms. The tendency to equip vehicles with advanced and embedded devices such as diverse sensors increases the capabilities of vehicles to provide computation and collection of multimedia content in the form of the vehicular network. Then, the taxonomy of cloud-based vehicular networks is addressed from the standpoint of the service relationship between the cloud computing and vehicular networks. In this paper, we identify the main considerations and challenges for cloud-based vehicular networks regarding multimedia services identified and propose potential research directions to make multimedia services achievable. More specifically, the authors quantitatively evaluate the performance metrics of these researches. For example, in the proposed broadcast storm mitigation scheme for vehicular networks, the packet delivery ratio and the normalized throughput can both achieve about $90 \%$, making the proposed scheme a useful candidate for multimedia data exchange. Moreover, in the video uplink scenarios, the proposed scheme is favorably compared with two well-known schedulers, M-LWDF and EXP, with the performance much closer to the optimum. 
In [15], the authors proposed a novel threshold credit-based incentive mechanism (TCBI) based on the modified model of population dynamics to efficiently resist the node compromise attacks, stimulate the cooperation among intermediate nodes, maximize vehicular nodes' interest, and realize the fairness of possessing the same opportunity of transmitting packets for credits. Then, a TCBI-based privacypreserving packet forwarding protocol was proposed to solve the open problem of resisting layer-adding attack by outsourcing the privacy-preserving aggregated transmission evidence generation for multiple resourceconstrained vehicles to the cloud side from performing any one-way trapdoor function only once. The vehicle privacy is well protected from both the cloud and transportation manager. Finally, formal security proof and the extensive simulation show the effectiveness of their proposed TCBI in resisting the sophisticated attacks and the efficiency in terms of high reliability, high delivery ratio, and low average delay in cloud-assisted vehicular DTNs.

In [16], the authors classify and describe the most relevant vehicular propagation and channel models, with a particular focus on the usability of the models for the evaluation of protocols and applications. First, they classify the models based on the propagation mechanisms they employ and their implementation approach. They also classify the models based on the channel properties they implement and pay special attention to the usability of the models, including the complexity of implementation, scalability, and the input requirements (e.g., geographical data input). They also discuss the less-explored aspects in vehicular channel modeling, including modeling specific environments (e.g., tunnels, overpasses, and parking lots) and types of communicating vehicles (e.g., scooters and public transportation vehicles). They conclude their paper by identifying the under researched aspects of vehicular propagation and channel modeling that require further modeling and measurement studies.

\section{Proposed new clustering mechanism}

Considering the above issues, an envisioned Integrated VANET-cellular networks Heterogeneous Wireless Network architecture is proposed. The architecture is depicted in Fig. 1. The scenario in this paper depicts a highway with a dual carriageway consisting of two lanes in each direction. With regard to placement, a GPS device was installed in all vehicles. Vehicles connect to different signal strengths on the road. New gateway is elected before the serving gateway loses its optimality to avoid the delay that occurs during the process of handoff.

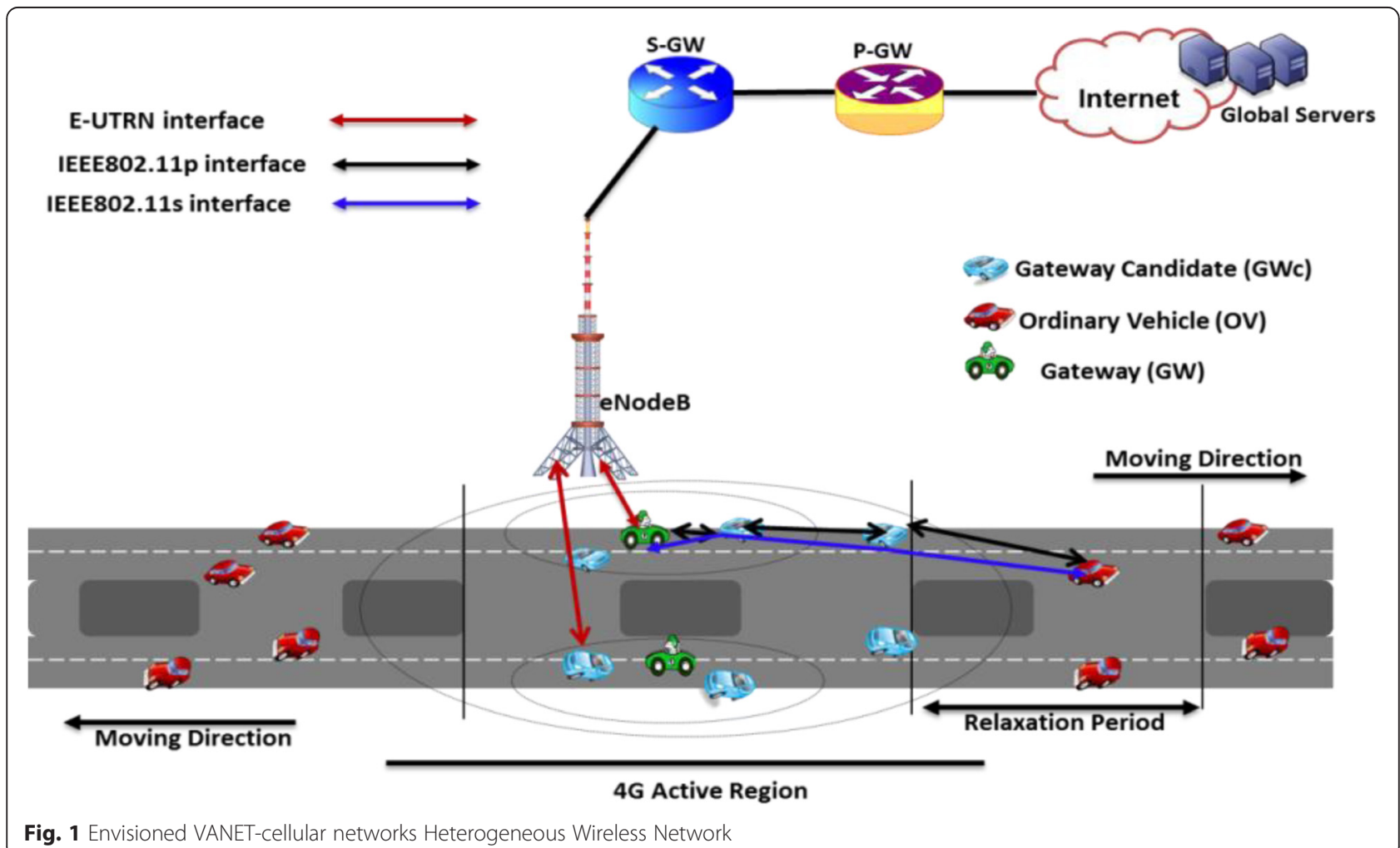

Fig. 1 Envisioned VANET-cellular networks Heterogeneous Wireless Network 
The connection between VANET nodes is multi-hop and is based on peer to peer.

The proposed clustering mechanism is employed upon the available GWcs (vehicles inside the converge zone and equipped with dual interface) cluster. The proposed algorithm1 is multi-metric algorithm, based on the Simple Additive Weighting (SAW) technique is used for gateway selection and for outranking the optimum gateway node. Three metrics are used for mobile gateway selection: LTE RSS $\left(x_{1}\right)$, available route capacity $\left(x_{2}\right)$, and stability of the link $\left(x_{3}\right)$. The stability of the link is defined by two metrics between the source and the $\mathrm{CH}$ : (1) Link Expiration Time (LET) and (2) Route Expiration Time (RET). LET is the link lifetime between the two adjacent vehicles and the minimum lifetime between the vehicles to select gateway which is referred to as Route Expiration Time (RET); the information of each metrics of each $\mathrm{CH}$ lies with the GWcs of the cluster. According to the given fact, the hybrid gateway discovery mechanism is employed.
E-HWMP is standard for wireless access to vehicular environments (WAVE) that employs a vehicular connection in vehicles (V2V). A Vehicle-to-Infrastructure (V2I) connection is not supported by the standard for wireless access. The Evolved Universal Terrestrial Radio Access Network (E-UTRAN) interface is utilized by the VANET to connect to the internet through eNodeB. Gateway numbers are required to be lower. The proposed scheme is cluster based. The clusters are formulated only at a certain point of the 3GPP LTE cell's edges when the received signal strength (RSS) crumbles to less than assured threshold.

This proposed scheme adopts a hybrid [2] that is a combination of both proactive and the reactive approaches. Firstly, the gateway broadcasts the Gateway Advertisement message (GANN) proactively to an area confined to a limited number of hops which is known as the TTL value of the message. The TTL is set to advertisement zone so that the advertisement message can be forwarded only up to this maximal number of hops through the ad hoc network. This area is known as the proactive zone. The nodes that are

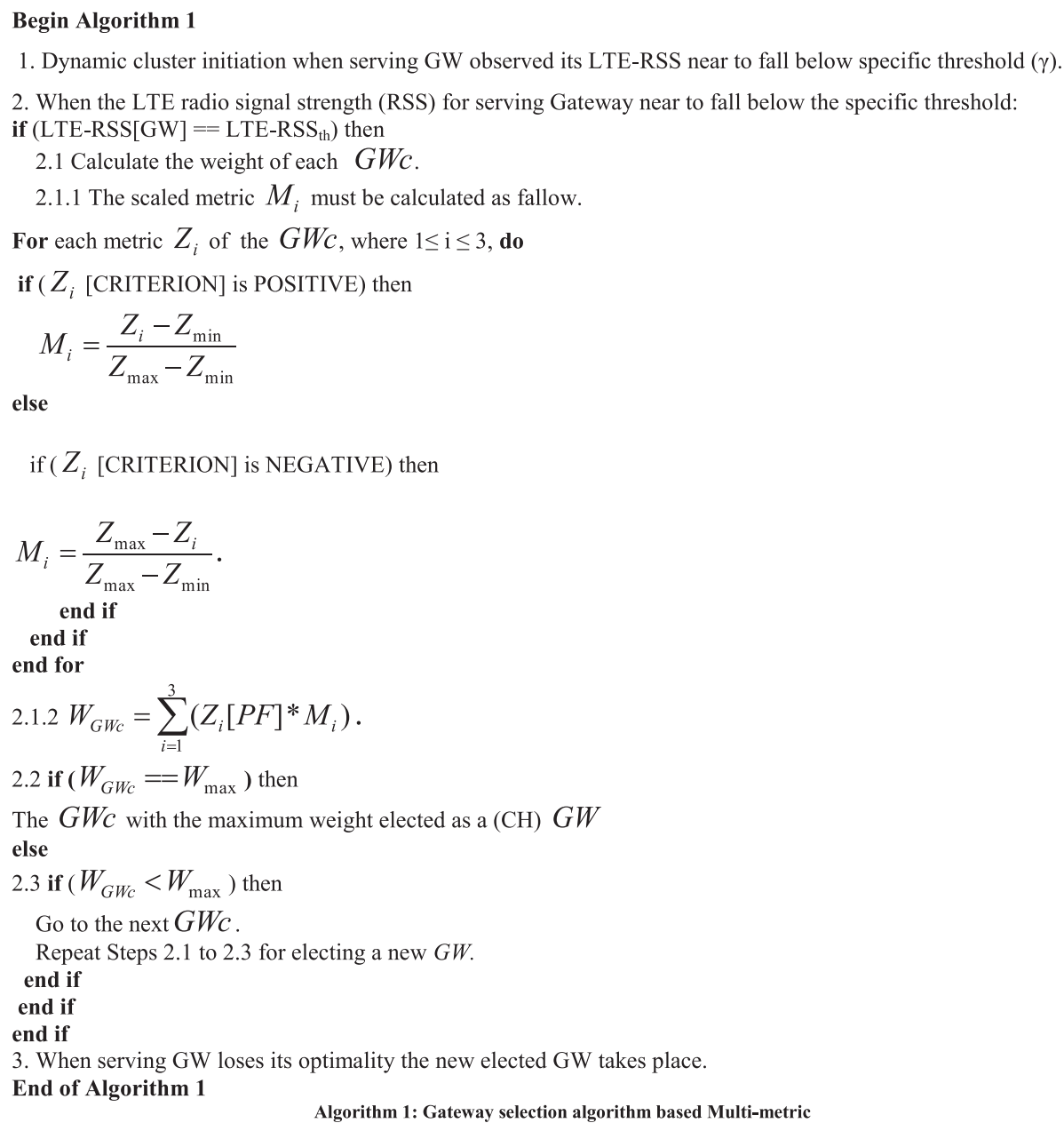




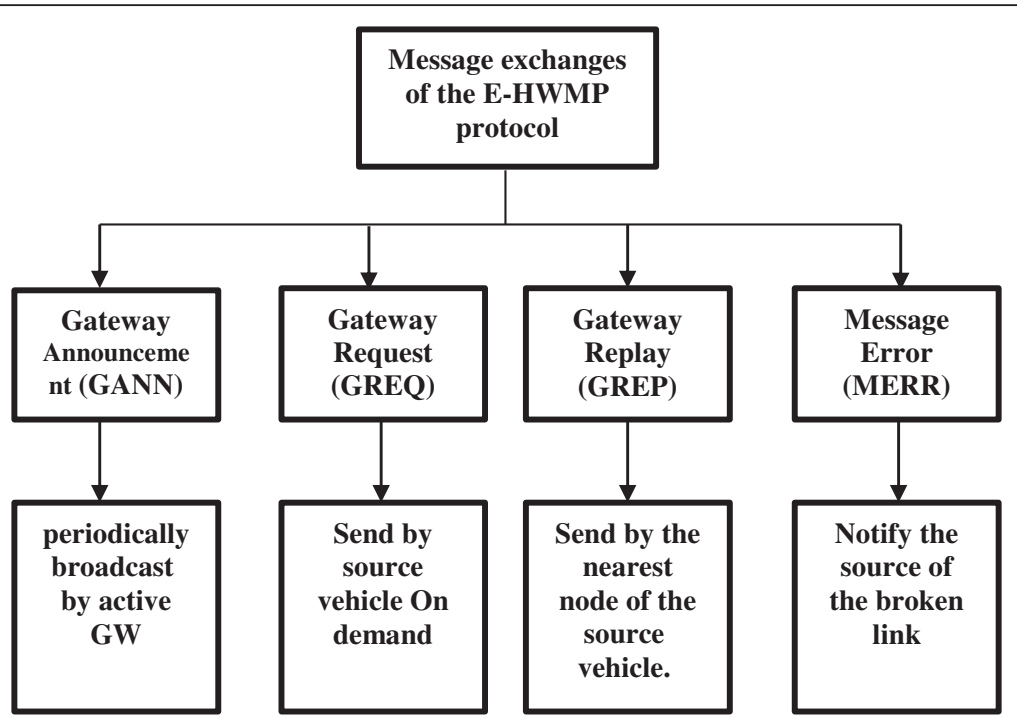

Fig. 2 Message exchanges of the E-HWMP protocol

outside the proactive zone establish connection to the gateway reactively, On-demand Broadcast of Gateway Request message (GREQ), by Active Sources in VANET.

In the hybrid approach, the gateway does always send a GWADV message every $5 \mathrm{~s}$. The main purpose of this approach (hybrid gateway discovery mechanism) is to the control overhead due to the reactive discovery scheme when there are many source nodes in the VANET. This approach also aims to reduce the overhead of the proactive protocols when the number of gateways increases.

Architecture of our proposed mechanism implemented using E-HWMP protocol. In Fig. 2, message exchanges of the E-HWMP protocol are shown.

\subsection{Simulation tools}

NS2.35 and VanetMobiSim-1.1 are the tools which are used in our simulation. Both are open source applications and are described in more detail below.

We chose VanetMobiSim in our simulation because it supports both micro-mobility and macro-mobility models

Table 1 Scenario Mobility features

\begin{tabular}{ll}
\hline Parameters & Value \\
\hline Type of road & Highway \\
Topology size & $8000 \times 1000$ \\
Number of lanes & $\begin{array}{l}\text { 2 lanes for each direction, vehicle move in } \\
\text { opposite direction } \\
\text { Varies between } 5 \text { to } 50 \mathrm{~m} / \mathrm{s} \text { and from } 18 \text { to } \\
\text { Maximum speed }\end{array}$ \\
Number of. & $\begin{array}{l}\text { Varies between } 10,30,50 . \\
\text { Simulation period }\end{array}$ \\
Stop time & Simulation period $500 \mathrm{~s}$ \\
\hline
\end{tabular}

Table 2 Simulation parameters

\begin{tabular}{ll}
\hline Parameters & Value \\
\hline Area & $8000 \times 1000$ \\
Channel & Channel/wireless channel \\
Propagation model & Propagation/TwoRayGround \\
Network interface & Phy/WirelessPhyExt \\
Mac interface & Mac/802 11Ext \\
Peak wireless transmission range & 300 m \\
Seed & 0.0 \\
Interface queue type & Queue/DropTail/PriQueue \\
Interface queue length & 20 packets \\
Antenna type & Antenna/OmniAntenna \\
Routing protocol & E-HWMP \\
Total number of VANET vehicles & Varies between 10, 30, 50 \\
Applications & FTP \\
Peak mobility speed & 50 ms - 1 \\
Mobility model & VanetMobiSim \\
Simulation stop & 500 \\
LTE RSS threshold & -94 dBM \\
Uplink frequency & 1.925 \\
Downlink frequency & 2.115 \\
Peak E-UTRAN UL channel bit rate & 5.2 \\
Peak E-UTRAN DL channel bit rate & 10.3 \\
Transmission range of eNodeB & 10 \\
Node B interface queue length & 20 packets \\
\hline
\end{tabular}




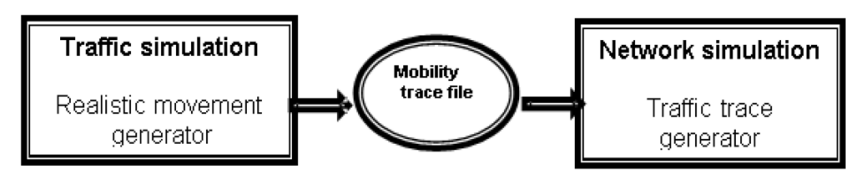

Fig. 3 Simulation architecture

$[17,18]$. This makes our simulation more realistic and covers up, as much as possible, the behavior of vehicular movement on the road. The result of the simulation is a trace file which can be imported in NS2 as a mobility file. VanetMobiSim mobility generator parameters are shown in Table 1. NS2 simulation parameters are shown in Table 2. The Simulation Architecture is shown in Fig. 3.

\subsection{Description of the simulations}

The simulation was performed by using a combination of VanetMobiSim and network simulators (NS2.35). A VanetMobiSim was used to simulate the mobility model $[17,19]$, while NS2.35 was used to measure the network performance [20] of the proposed mechanism.

We have implemented three scenarios in our simulation which will be demonstrated in the following sections. Table 1 shows mobility generator parameters. VanetMobiSim-1.1 scenarios are shown in Fig. 4.
Table 2 shows the parameter used to configure our network in ns2. Screenshots of the simulation scenarios are shown in Fig. 5.

\section{Results and analysis}

The proposed scheme was implemented in NS2.35. EHWMP routing protocol was used to enable communication between the vehicles. The number of vehicles ranges between 10,30 , and 50 . The transmission range of vehicles' wireless devices was kept constant. Thirtysix kilometer per hour and $180 \mathrm{~km} / \mathrm{h}$ were used respectively as the minimum and maximum speeds for the vehicles. The performance of our proposed scheme was evaluated in terms of packet delivery ratio, packet lost, throughput, overhead, and average end-to-end delay.

In Fig. 6, it is observed that the packet delivery ratio of our scheme is higher for lower speed of the vehicles but decreases when the speed of the vehicles is increased. Also, it is observed that the packet delivery

\section{$\otimes \Theta($ VanetMobisim}

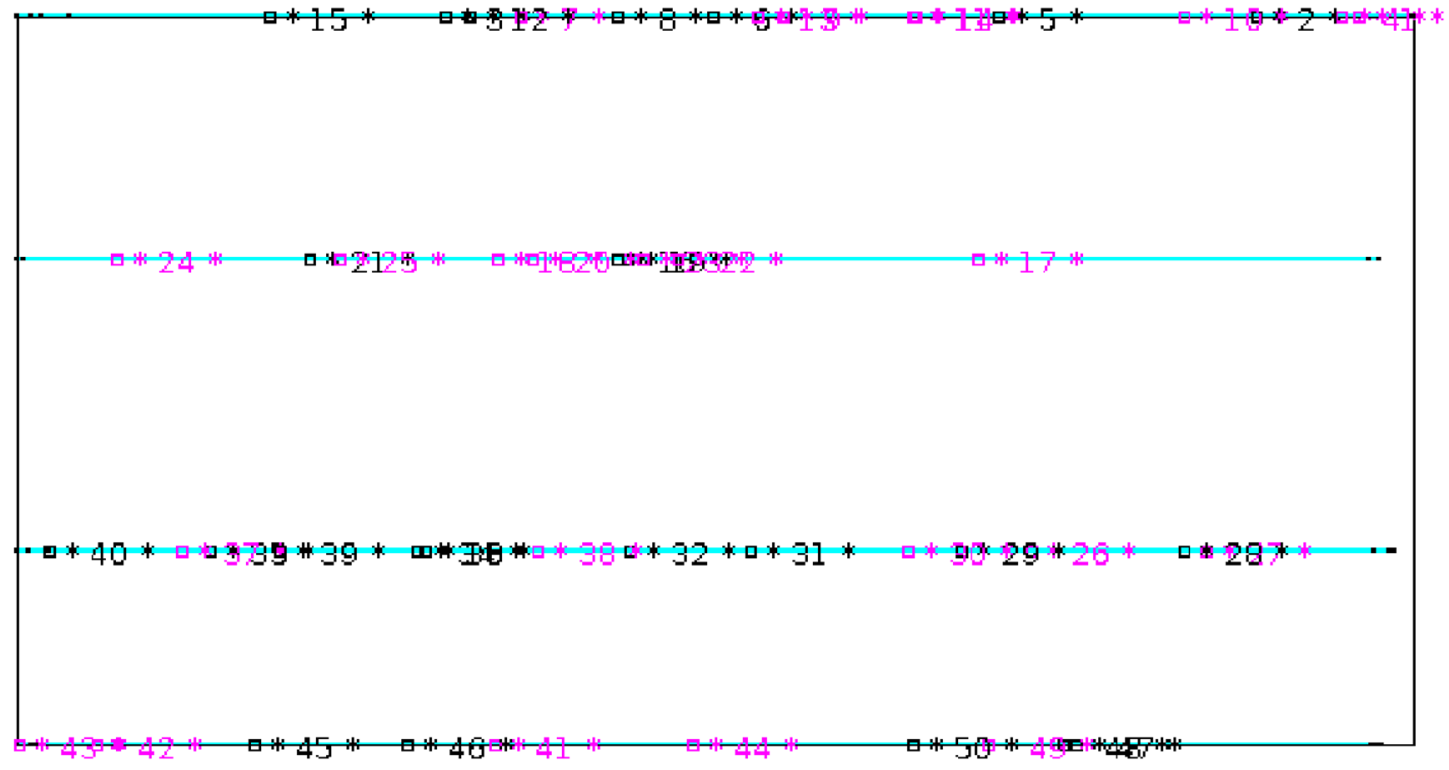

Simulation time: 347.187 s Pointer: \{ 521 570\}

Fig. 4 Vehicles mobility model for 50 vehicles on the highway 


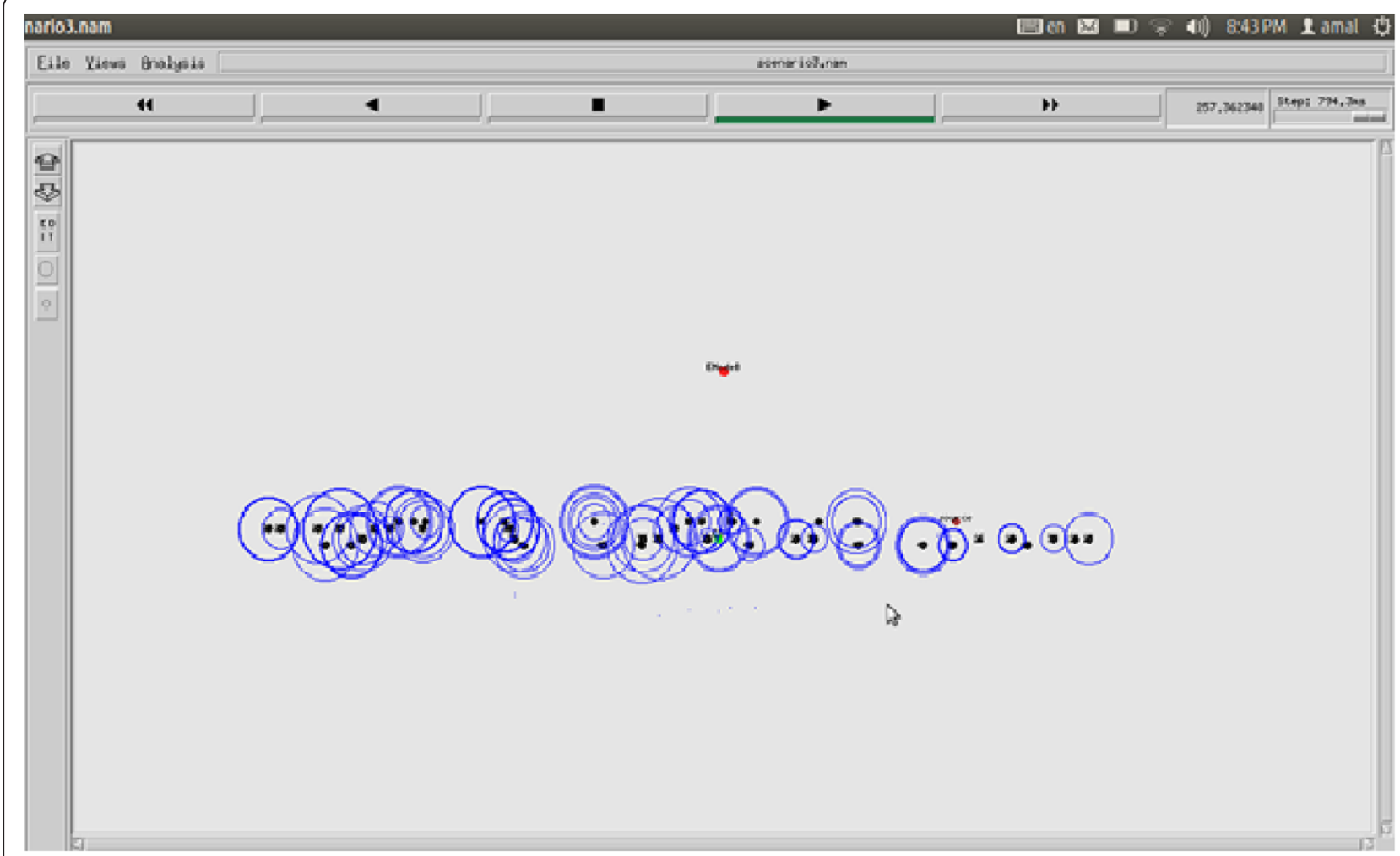

Fig. 5 Simulation scenario with 50 vehicles

ratio is higher when there is a large density of vehicles with low speed more than the small density of vehicles with low speed.

In Fig. 7, it is observed that the packet drop ratio of our mechanism is increased in high-speed vehicles, but the drop ratio of our scheme decreases when the speed of the vehicles decrease. Also, it is observed that the packet drop ratio is lower when there is a large density

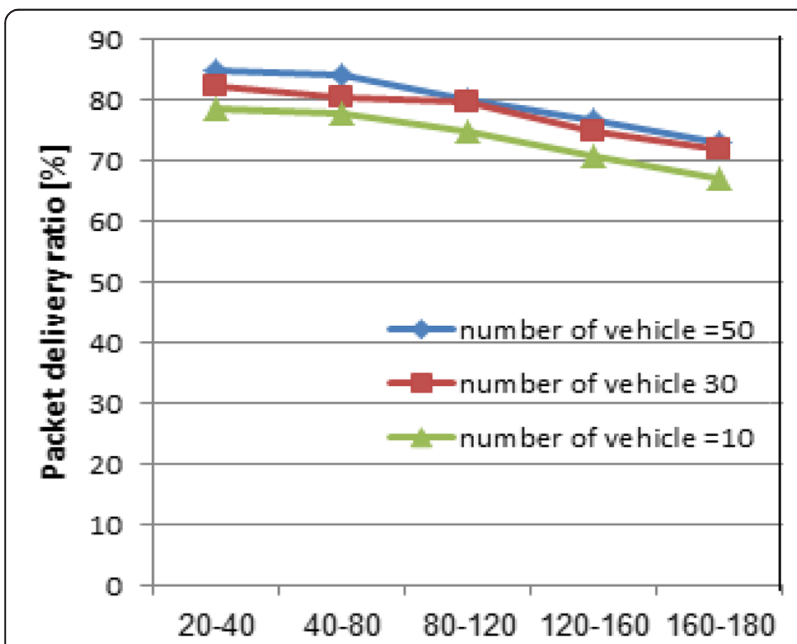

Fig. 6 Packet delivery ratio of our scheme with E-HWMP routing protocol with a variation in mobility speed of vehicles of vehicles with low speed more than the small density of vehicles with low speed.

In Fig. 8, it is observed that the gateway selection delay of our scheme increases for a small number of vehicles and decreases when the density of the vehicles is increased.

It can be seen from Fig. 9 that the average throughput of our scheme is increased for a large number of vehicles

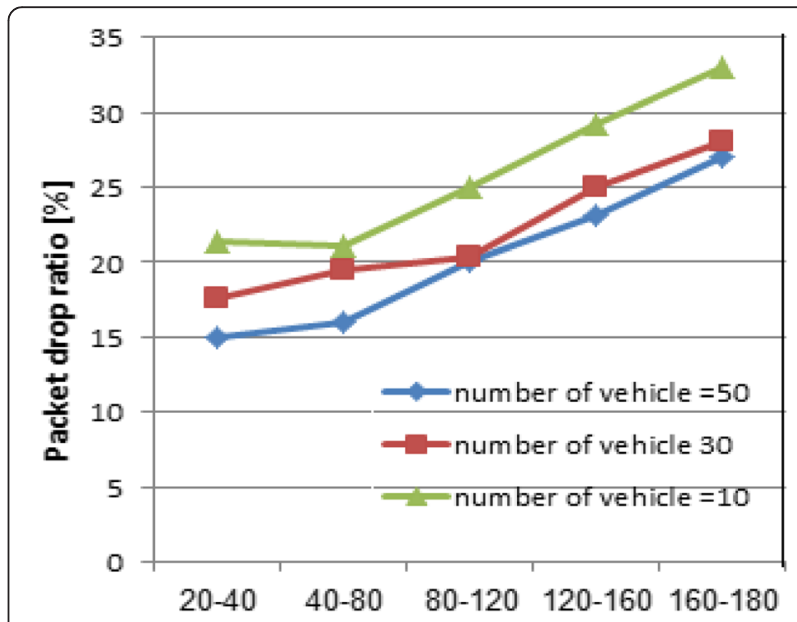

Fig. 7 Packet drop ratio of our scheme with E-HWMP routing protocol with a variation in mobility speed of vehicles 


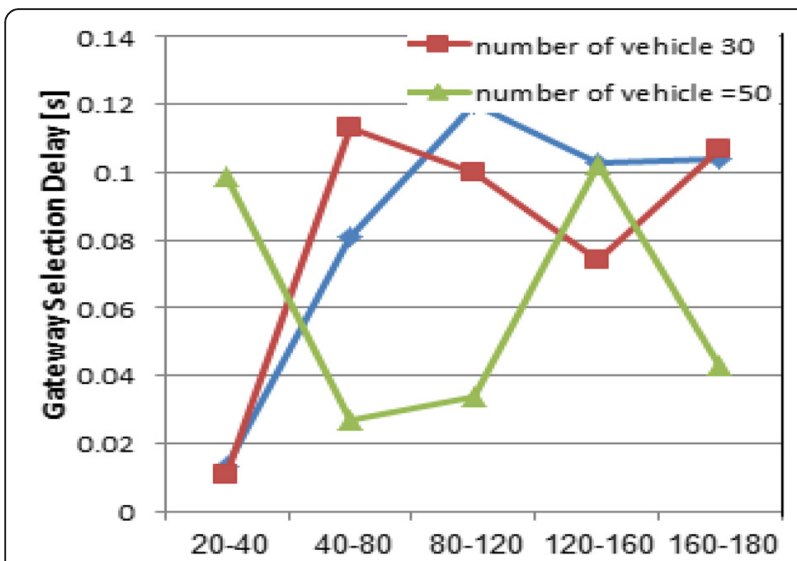

Fig. 8 Gateway selection delay of our scheme with E-HWMP routing protocol with a variation in mobility speed of vehicles

but when the number of the vehicles decrease, the throughput of our scheme decreases.

Figure 10 shows that our proposed mechanism performs well in terms of lower packet drop ratio compared to CMGM mechanism and SGS schemes. Our proposed E-HWMP mechanism shows a $16.2 \%$ improvement over SGS with AODV and a $19.5 \%$ over CMGM over AODV. It should be noted that the reason for this is the fact that in our mechanism, all vehicles inside the coverage zone are connected directly to the E-node B, so that the frequent network disconnection is reduced and E-HWMP helps any source vehicle to deliver its packet to E-node $B$ when the vehicles are outside the coverage zone. Regardless of the underlying protocol, the number of sources increases, the packet drops, subsequently increases, especially when the gateway is on the verge of losing its optimality.

Figure 11 illustrates that our proposed mechanism achieves a good performance in terms of less control

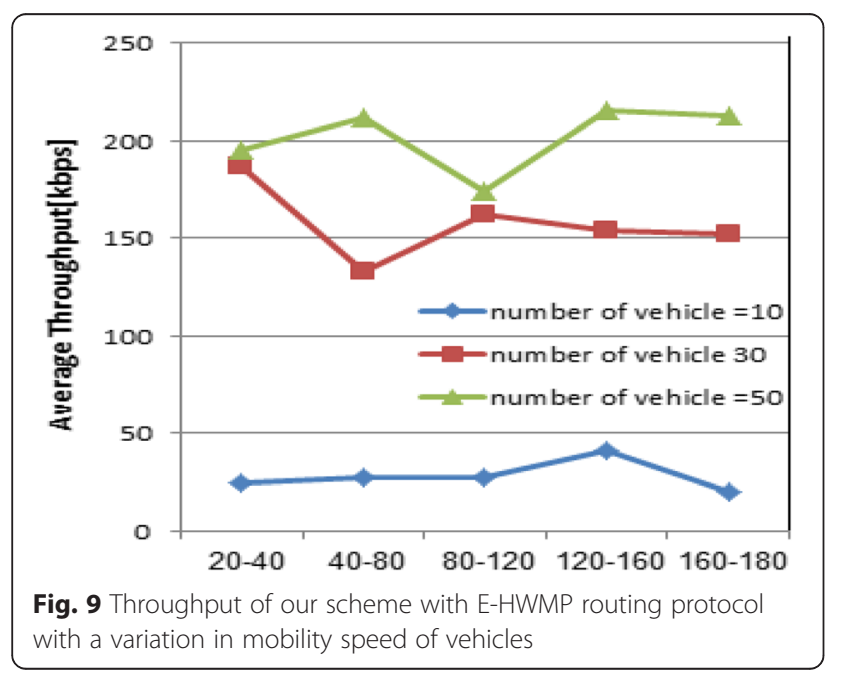

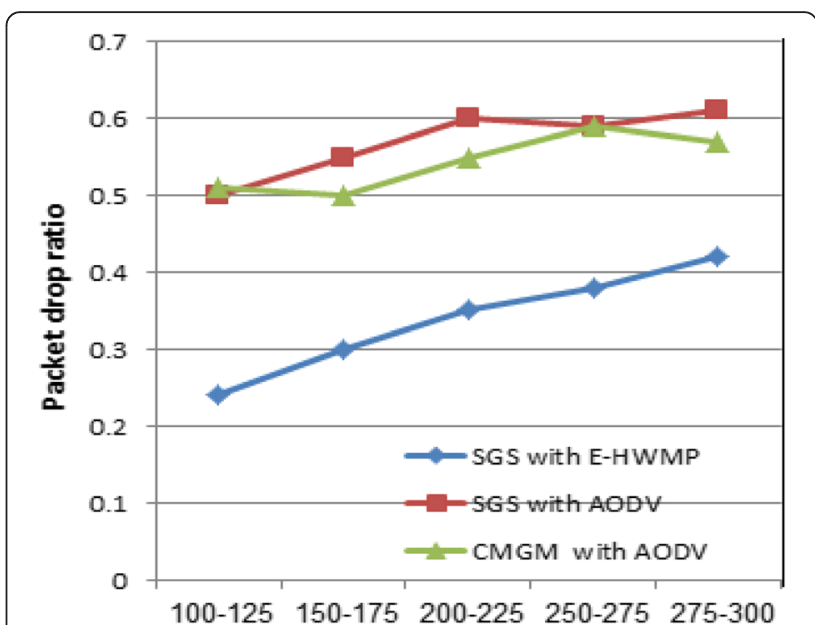

Fig. 10 Packet Drop ratio of SGSS with E-HWMP, as well as AODV and CMGM with AODV that are in a range of Variation of IEEE802.11 transmission range of vehicles

packet overhead, compared to the CMGM and SGS schemes. Our proposed E-HWMP mechanism shows a $4.5 \%$ improvement in reducing control packet overhead compared to SGS over AODV and a $10.15 \%$ improvement compared to CMGM over AODV.

Figure 12 shows that our proposed mechanism performs well in terms of higher packet delivery ratio compared to the CMGM and SGS schemes. Our proposed E-HWMP mechanism shows a $20.4 \%$ improvement in terms of packet delivery ratio over SGS with AODV and a $28.7 \%$ improvement over CMGM over AODV. It should be noted that the reason for this is the fact that in our mechanism, all vehicles inside the coverage zone are connected directly to the E-node B so that the frequent network disconnection was reduced and

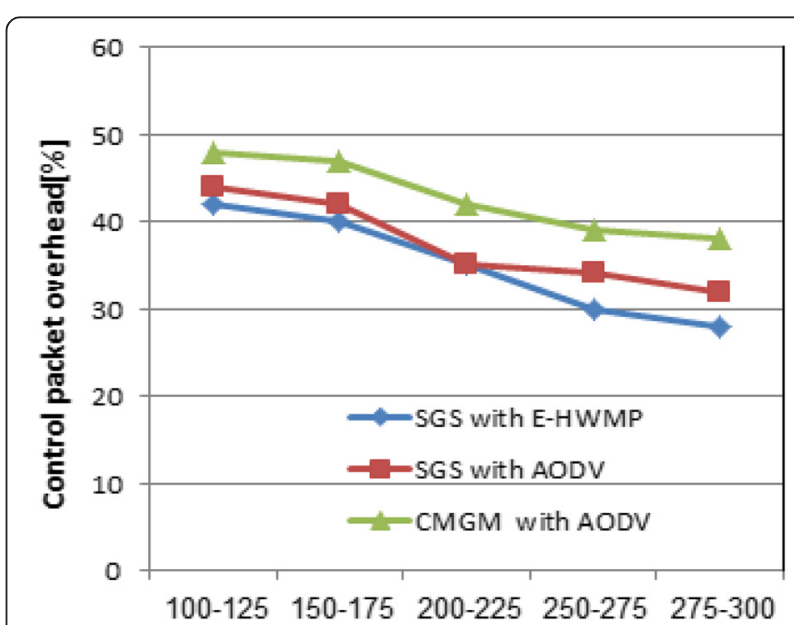

Fig. 11 Control packet overhead of SGSS with E-HWMP, as well as AODV and CMGM with AODV that are in a range of variation of IEEE802.11 transmission range of vehicles 


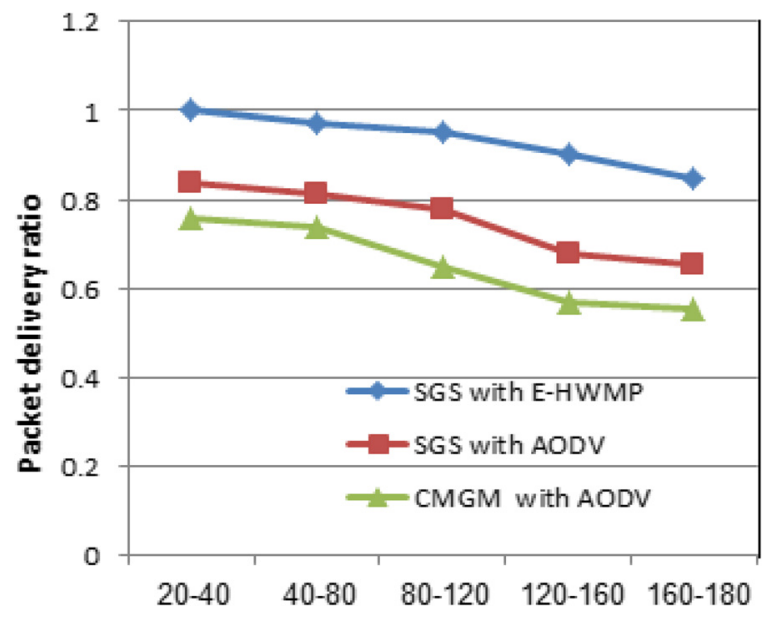

Fig. 12 Packet delivery ratio of SGSS with E-HWMP, as well as AODV and CMGM with AODV that are in a range of Variation of IEEE802.11 transmission range of vehicles

E-HWMP helps any source vehicle to deliver its packet to E-node B when the vehicles are outside the coverage zone.

In Fig. 13, it is observed that our proposed mechanism achieves a good performance in terms of less delay compared to the CMGM and SGS schemes. Our proposed E-HWMP mechanism exhibits $10.3 \%$ less delay compared with SGS over AODV and $28.71 \%$ compared with CMGM over AODV. It can be pointed out that the reason for this is the fact that in our mechanism, all vehicles inside the coverage zone are connect directly to the E-node $\mathrm{B}$ so that the frequent network disconnection was reduced and E-HWMP helps any source vehicle to deliver its packet to E-node B when the vehicles are outside the coverage zone.

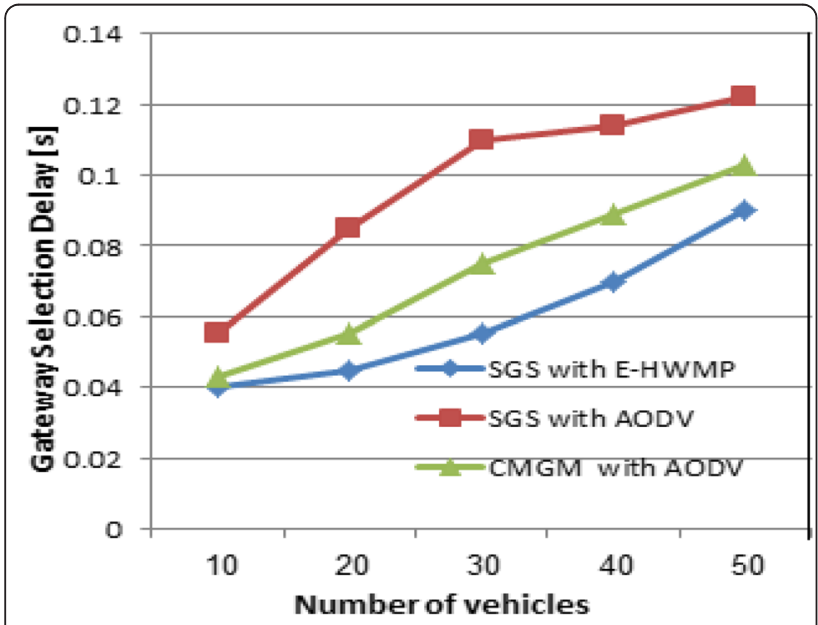

Fig. 13 Gateway Selection Delay vs. different numbers of vehicles in the VANET

\section{Conclusions}

In this paper, a new network structure is introduced for integration of VANET and cellular network. This is done to enhance the heterogeneity in vehicular communication, to reduce the amount of overhead, increase the packet delivery, throughput, reduce the average end-to-end delay, and reduce the packet drop ratios in multi-hop VANET and cellular network with high traffic data transactions. A few numbers of adequate vehicles are selected to serve as gateways between VANET and cellular network. This paper also analyzed the performance and compared the proposed mechanism with existing mechanisms used for a vehicular ad hoc network. We also introduced a new mobility model in which the relationship between vehicle density, speed, and the follow-on distance rule is derived. The performance of the overall architecture is evaluated using computer simulations in NS2.35.

\section{Competing interests}

The authors declare that they have no competing interests.

\section{Author details}

${ }^{1}$ Electronics Engineering School, Sudan University of Science and Technology (SUST), Khartoum, Sudan. ${ }^{2}$ IBM India Private Limited, Kolkata, India.

${ }^{3}$ Department of Electrical and Computer Engineering, International Islamic

University Malaysia (IIUM), 53100 Kuala Lumpur, Malaysia.

Received: 7 April 2016 Accepted: 5 July 2016

Published online: 15 July 2016

\section{References}

1. A Joshi, P Sirola, KC Purohit. Comparative Study of Enhanced AODV Routing Protocols in VANET. Int. J. Comput. Appl. (0975-8887). 96(18), June 2014, pp. 22-27.

2. AA Eltahir, RA Saeed, "Performance Evaluation of an Enhanced Hybrid Wireless Mesh Protocol (E-HWMP) Protocol for VANET", International Conference on Computing, Control, Networking, Electronics and Embedded Systems Engineering (ICCNEEE 2015), 2015, pp. 95-100

3. MA Alawi, RA Saeed, AA Hassan, RA Alsaqour, Simplified gateway selection scheme for multi-hop relay vehicular ad hoc network. Int. J. Commun. Syst. 27(12), 3855-3873 (2014)

4. A. Benslimane, T. Taleb, R. Sivaraj, Dynamic clustering-based adaptive mobile gateway management in integrated VANET-3G heterogeneous wireless networks. IEEE. J. Selected Areas Commun. 29(3), pp. 559-570

5. C Kathirvel, D Loyd, Optimized Hybrid Wireless Mesh Protocol using Estimation of Paket Loss Rate Algorithm for VANET. International Journal of Computer Science and Mobile Applications 2(3), 117-123 (2014)

6. AA Eltahir, RA Saeed, MA Alawi, "An enhanced hybrid wireless mesh protocol (E-HWMP) protocol for multihop vehicular communications" 2013 International Conference on Computing, Electrical and Electronics Engineering (ICCEEE), 2013, pp. 1-8

7. R Sivaraj, AK Gopalakrishna, Qos-enabled group communication in integrated VANET-LTE heterogeneous wireless networks, IEEE 7th International Conference on Wireless and Mobile Computing, Networking and Communications (WiMob), 2011

8. J Zhao, T Arnold, Y Zhang, G Cao, Extending DriveThru Access by Vehicle-toVehicle Relay, 2008. ACM VANET

9. T Meng et al., Spatial Reusability-Aware Routing in Multi-Hop Wireless Networks. IEEE TC 65, 244-255 (2016)

10. XM Zhang et al., Interference-based topology control algorithm for delayconstrained mobile ad hoc networks. IEEE Trans. Mob. Comput. 14(4), 742754 (2015)

11. P Li et al., Reliable multicast with pipelined network coding using opportunistic feeding and routing. IEEE Trans. Parallel Distrib. Syst. 25(12), 3264-3273 (2014) 
12. $Y$ Zeng et al., Directional routing and scheduling for green vehicular delay tolerant networks. Wirel. Netw 19(2), 161-173 (2013)

13. L Zhou et al., Distributed media services in P2P-based vehicular networks. IEEE T. Vehicular Technology 60(2), 692-703 (2011)

14. M-K Jiau et al., Multimedia services in cloud-based vehicular networks. IEEE Intell. Transport. Syst. Mag. 7(3), 62-79 (2015)

15. JN Zhou et al., Seure and privacy preserving protocol for cloud-based vehicular DTNs. IEEE Trans. Inf. Forensics Secur. 10(6), 1299-1314 (2015)

16. W Viriyasitavat et al., Vehicular communications: survey and challenges of channel and propagation models vehicular technology magazine. IEEE 10(2), 55-66 (2015)

17. J. Haerri, M. Fiore, F. Fethi, C. Bonnet, VanetMobiSim: generating realistic mobility patterns for VANETs. Institut Eurécom and Politecnico Di Torino, 2006

18. O. Alsaqour, R. Alsaqour, T. Alahdal, R.A. Saeed, M. Al-Hubaishi, "A comparative study of simulation based performance evaluation of routing protocol for ad-hoc networks", Innovations and Advances in Computing, Informatics, Systems Sciences, Networking and Engineering, Springer International Publishing, 2015

19. CANU Research Group (Stuttgart University) http://vanet.eurecom.fr/

20. ns, "ns2", http://www.isi.edu/nsnam/ns/index.html, Accessed 22 Dec 2015.

\section{Submit your manuscript to a SpringerOpen ${ }^{\odot}$ journal and benefit from:}

- Convenient online submission

- Rigorous peer review

- Immediate publication on acceptance

- Open access: articles freely available online

- High visibility within the field

- Retaining the copyright to your article

Submit your next manuscript at $\boldsymbol{s p r i n g e r o p e n . c o m ~}$ 